\title{
A SIMPLE EXAMPLE OF TWO DIMENSIONAL MODEL FOR TRAFFIC: DISCUSSION ABOUT ASSUMPTIONS AND NUMERICAL METHODS
}

\section{Stephane Mollier}

Univ. Grenoble Alpes, CNRS, Inria, Grenoble INP, GIPSA-Lab, 38000 Grenoble, France, Email: stephane.mollier@gipsa-lab.fr

\section{Maria Laura Delle Monache, Corresponding author}

Univ. Grenoble Alpes, Inria, CNRS, Grenoble INP, GIPSA-Lab, 38000 Grenoble, France , Email: ml.dellemonache@inria.fr

\section{Carlos Canudas-de-Wit}

Univ. Grenoble Alpes, CNRS, Inria, Grenoble INP, GIPSA-Lab, 38000 Grenoble, France, Email: carlos.canudas-de-wit@gipsa-lab.fr

Word Count: 4668 words, 9 figures, 1 table $=7168$ words

Submission Date: November 10, 2017 
1 ABSTRACT

2 This article presents a two dimensional macroscopic model for traffic flow on a network. We con3 sider a two dimensional conservation law where we suggest a new method to estimate the direction 4 of the velocity using only information and properties of the network. We present an algorithm for 5 the computation of the two dimensional velocity on the network and present numerical simula6 tions. Moreover, the numerical approximation of the two dimensional equation is carried out using 7 an operator and splitting method and we present the results of some simulations on some realistic 8 network. Lastly, we propose a comparison with the well-known CTM method.

9 Keywords: Two dimensional macroscopic model, traffic flow, partial differential equations 10 (PDEs), dimensional splitting, dense urban network. 


\section{INTRODUCTION}

2 This paper deals with the problem of designing two-dimensional macroscopic traffic flow models

3 for large-scale traffic networks. Traffic modeling literature is rather vast (see van Wageningen-

4 Kessels et al. (1) for a complete description of traffic models evolution). A key observation in

5 traffic flow modeling is that the speed of vehicles can be expressed as a function of vehicle den-

6 sity, see Greenshields et al. (2). The flux-density relation is well known as Fundamental Diagram

7 (FD), and used as a key element in macroscopic traffic model design. Macroscopic models were

8 introduced by Lighthill, Whitham and Richards (LWR) Lighthill and Whitham (3)Richards (4).

9 The LWR is described by a partial differential equation and it is able to reproduce several features

10 that are observed in traffic data on highway like shock and rarefaction waves. The discretized ver-

11 sion of the LWR is known as the Cell Transmission Model (CTM), see Daganzo (5). Models of

12 junction Coclite et al. (6) together with some split ratio assumptions allow to extend the CTM and other similar models to urban networks. Those junction models ensure a correct propagation of flux locally through an intersection, and together with the CTM can be used to describe 1-D flow propagation along the network. Modeling each specific road in a large and complex network may be tedious, difficult to tune, and in some cases computationally expensive. These difficulties have motivated the research for aggregated traffic models describing the traffic evolution by geographical zones rather than modeling each individual road and intersection in the city network. A first natural approach has been to extend the 1-D FD to an urban area. This is called the Macroscopic Fundamental Diagram (MFD), which captures the aggregated number of vehicles in a given area (density/occupancy), and maps it into their respective averaged velocity (flows). The existence of the MFD have been studied first analytically by Daganzo and Geroliminis (7), then numerically by Geroliminis and Sun (8), and lastly using real field data by Geroliminis and Daganzo (9). The MFD computed in that way, is then used in the cumulative mass conservation equation

$$
\frac{d n}{d t}(t)=\Phi_{\text {in }}(t)-\Phi_{\text {out }}(n(t)), \quad \forall t \in \mathbb{R}^{+}
$$

where $n$ represent the cumulative number of car in the considered area, $\Phi_{\text {in }}$ and $\Phi_{\text {out }}$ are the area inflow and outflow, respectively. In this set-up, the inflow $\Phi_{i n}$ is assumed to be known (or measured), whereas the outflow $\Phi_{\text {out }}$ is computed using the MFD and the assumed known user trip length. These types of model focus on the modeling of the number of vehicles in an area with a single variable. Then, they preserve less information about the spatial distribution of vehicle than models which consider the density. However, Hajiahmadi et al. (10) Leclercq et al. (11) investigate the extension to multi-zone model to consider different MFD for each zone for which the road pattern may differs. It is worth to notice that these models are set once the modeling zone has been defined, but they are not able to "expand/contract" themselves so that the modeling zone can not be adapted to the changes of traffic conditions.

Another approach to model traffic in large networks is the two dimensional models (2D model) where the density depends on 2D spatial and temporal coordinate systems: a point in the plane $(x, y)$, and time $t$. The first researchs on 2D traffic modeling have been done in the static case i.e. without time dependency Beckmann (12). Contrary to dynamical model which target to predict the evolution of the system, these static model are interested in some traffic feature as for example, optimal assignment of traffic. An overview of existing static 2D models are given in Ho and Wong (13). In this paper, we will focus on 2D dynamical models. The motivation of such models is to simplify the number of individual variables, avoid specific models for junctions, and simplify the 
1 tuning of the model parameters. There is also some motivation for simplifying both the control

2 design and the use of the model for predicting the density evolution. Such models can take the 3 following general form:

$$
\left\{\begin{array}{c}
\frac{\partial \rho(t, x, y)}{\partial t}+\nabla \cdot \vec{\Phi}(\rho(t, x, y), \theta(x, y))=0 \\
\rho(0, x, y)=\rho_{0}(x, y)
\end{array}, \quad \forall t \in \mathbb{R}^{+}, \forall(x, y) \in \Omega\right.
$$

5 where $\rho$ is the aggregated density , $\Phi$ the flow defined as the product of the density $\rho$ and velocity $6 v$ and $\theta$ the velocity direction. This type of model based on a two-dimensional conservation laws 7 are also used in pedestrian modeling which started with the work of Helbing (14), Hughes (15). 8 Several extensions for these pedestrian models have been considered including second order model 9 Jiang et al. (16) and non-local flux Colombo et al. (17). A numerical comparison of pedestrian 10 models can be seen in Twarogowska et al. (18) and a review of some of these models are given in 11 Kormanová et al. (19).

12 The literature concerning two-dimensional traffic models is not broad, only few works focused on 13 2-D traffic flow models, see Della Rossa et al. (20), Jiang et al. (21), Du et al. (22), Jiang et al. (23), 14 Romero Perez and Benitez (24), Saumtally (25), Sossoe and Lebacque (26), Chetverushkin et al. (27), Sukhinova et al. (28). We assume for 2D model that vehicles run on a dense network instead of a single road. The key point of the 2-D models is to understand how the FD can be translated for 2-D traffic. First of all, the FD in 1D holds on the assumption that the velocity is a function of only the density allowing then the construction of a unique curve that relates traffic flux and traffic density. In 2D the problem is more complex and in the literature several approaches have been proposed. In Romero Perez and Benitez (24), an advection diffusion equation is introduced and the authors assume that the traffic velocity does not depend on the density but it is instead a function of the space, i.e. the network. In this way the velocity is predetermined and the advectiondiffusion equation becomes linear. Other authors, like Della Rossa et al. (20) consider the velocity as a scalar function of the density and its direction is predetermined by estimating the shape of the network. In turn, they solve then a diffusion equation where the direction of traffic flow is indicated by the direction of the speed but also by the diffusion operator. In Jiang et al. (21), Du et al. (22), the authors consider a model inspired from pedestrian models in which the flux takes the direction of the lowest cost that they determine with the help of an Eikonal equation. Lastly in Jiang et al. (23), this model has been extended to a second order dynamics in order to have a more accurate acceleration model for pollutant estimation. In Saumtally (25) and Sossoe and Lebacque (26), they investigate the possibility of constructing a two dimensional model which take into account conflicts that occur on intersection. In this spirit, they take inspiration from models of junctions in one dimension as Lebacque and Khoshyaran (29). However, the problem is formulated in a discrete setting using the concepts of demand and supply. In Chetverushkin et al. (27) and in Sukhinova et al. (28), authors consider two dimensional models for traffic but in order to be applied to the case of multilane road instead of the case of road network.

The contribution of this article is to present a new traffic flow model which extends the LWR model in two dimensions. We suggest a way to estimate the direction of the velocity using information and properties from the network. We also describe entirely the numerical process based on operator and dimensional splitting and show some simulations.

The paper is organized as follows. In the first section, we introduce the model and the assumptions taken to construct this model. Then, we suggest a methodology to estimate the velocity fields for 
1 the model. Finally, we describe the numerical process, show the results of the simulations and

2 present a comparison between our two dimensional model and the CTM.

3 FROM TRAFFIC FLOW MODEL IN 1D TO 2D

4 Recall of 1D LWR model

5 In the fifties Lighthill, Whitham and independently Richards Lighthill and Whitham, Richards

$6(3,4)$ introduced a hydrodynamic model (LWR model) for traffic flow. This model is inspired from

7 fluid dynamics. The main assumption of this model is the hypothesis that the velocity $v \in\left[0, v_{\max }\right]$

8 depends only on the main traffic density $\rho \in\left[0, \rho_{\max }\right]$. The presence of this relation ensures the

9 existence of the Fundamental Diagram (FD) (2). The FD gives the link between the main traffic

10 density and the traffic flow, see Figure 1. In the LWR model the flux function is given by $\Phi(\rho)$ :

$11\left(0, \rho_{\max }\right) \rightarrow \mathbb{R}=v(\rho(t, x)) \rho(t, x)$.

12 The evolution in time of the main traffic density is given by the following scalar conservation law:

$$
\begin{cases}\frac{\partial \rho(t, x)}{\partial t}+\frac{\partial \Phi(\rho(t, x))}{\partial x}=0 & (t, x) \in \mathbb{R}^{+} \times \mathbb{R} \\ \rho(0, x)=\rho_{0}(x) . & x \in \mathbb{R}\end{cases}
$$

14 Where the velocity and the flux are given by

$$
v(\rho)=\left(1-\frac{\rho}{\rho_{\max }}\right) v_{\max }
$$

15

$$
\Phi(\rho)=\rho\left(1-\frac{\rho}{\rho_{\max }}\right) v_{\max }
$$

16 The flux $\Phi$ is concave and attains one maximum which we denote $\Phi^{\max }$ at $\rho_{\text {crit }}$. Moreover, $\Phi(0)=$ $17 \Phi\left(\rho_{\max }\right)=0$, see Figure 1(b).

18 Extension to conservation laws in two dimensions

In this section, we investigate how the LWR model can be extended to a two dimensional continuous model and what are the main differences.

It is possible to derive a two dimensional balance equation in a domain $\Omega \subset \mathbb{R}^{2}$ using the conservation of the mass. Let $A \subset \Omega$ be an arbitrary subdomain, $n_{\mathrm{A}}(t)$ the number the vehicle within $A$ at time $t$ and $n_{\mathrm{in}}^{\Delta \mathrm{t}}, n_{\text {out }}^{\Delta \mathrm{t}}$ the number of vehicles which respectively enter and exit $A$ between $t$ and $t+\Delta t$. Then:

$$
n_{\mathrm{A}}(t+\Delta t)-n_{\mathrm{A}}(t)=n_{\mathrm{in}}^{\Delta \mathrm{t}}-n_{\text {out }}^{\Delta \mathrm{t}}, \quad \forall t \in \mathbb{R}^{+}
$$

dividing by $\Delta t$ and passing at the limit when $\Delta t \rightarrow 0$, we obtain:

$$
\frac{\partial\left(n_{\mathrm{A}}(t)\right)}{\partial t}=\Phi_{\text {in }}(t)-\Phi_{\text {out }}(t), \quad \forall t \in \mathbb{R}^{+}
$$

where $\Phi_{\text {in }}$ the inflow and $\Phi_{\text {out }}$ the outflow of the subdomain A.

We define the density of vehicle $\rho(t, x, y): \mathbb{R}_{+} \times \Omega \rightarrow\left[0, \rho_{\max }\right]$ and the flux of vehicle $\vec{\Phi}(t, x, y, \rho): \mathbb{R}_{+} \times \Omega \times\left[0, \rho_{\max }\right] \rightarrow \mathbb{R}^{2}$. By definition of the density and the flux, we have:

$$
\frac{\partial}{\partial t}\left(\iint_{A} \rho(t, x, y) d x d y\right)=-\oint_{\partial A} \vec{\Phi}(t, x, y, \rho) \cdot \vec{n}(x, y) d l, \quad \forall t \in \mathbb{R}^{+}
$$




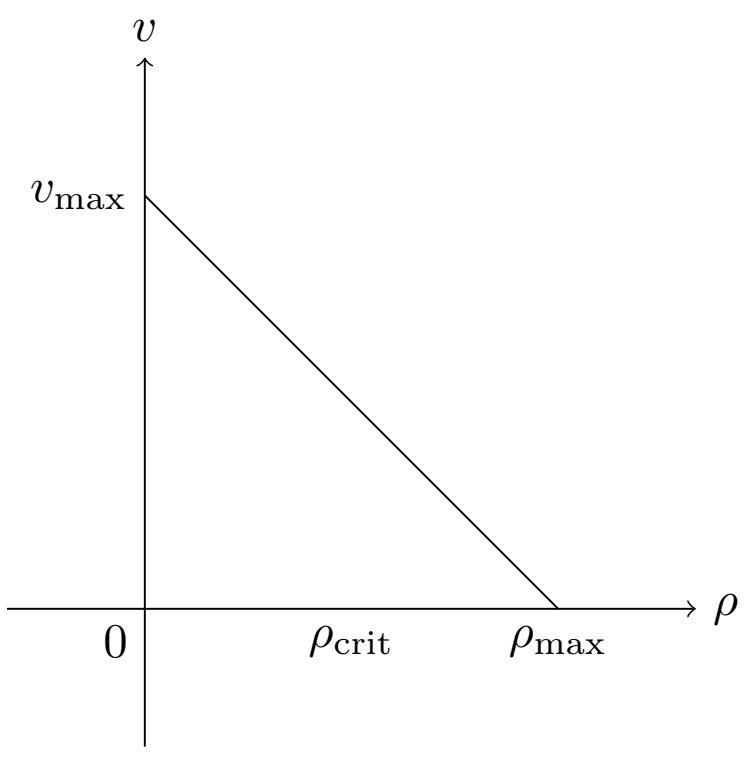

(a) Relation velocity density

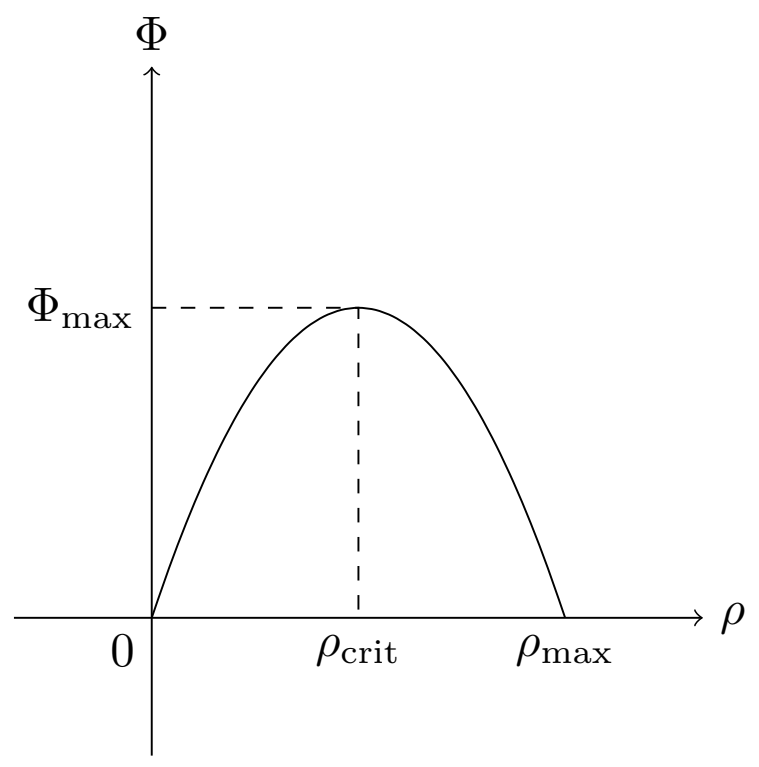

(b) Relation flux density:fundamental diagram (FD)

FIGURE 1 Speed and flow vs. density

1 where $\vec{n}$ is the vector normal to the boundary and $l$ the variable which follows the boundary of the 2 subdomain.

3 Thanks to the divergence theorem, we have:

$$
\iint_{A} \frac{\partial \rho(t, x, y)}{\partial t} d x d y=-\iint_{A} \nabla \cdot \vec{\Phi}(t, x, y, \rho) d x d y, \quad \forall t \in \mathbb{R}^{+} .
$$

4 The equality (9) hold for all $A \subset \Omega$, therefore we get:

$$
\frac{\partial \rho}{\partial t}(t, x, y)+\nabla \cdot \vec{\Phi}(t, x, y, \rho)=0, \quad \forall t \in \mathbb{R}^{+}, \forall(x, y) \in \Omega
$$

5 This equation is completed with the following initial condition:

$$
\rho(0, x, y)=\rho_{0}(x, y) . \quad \forall(x, y) \in \Omega
$$

6 For readers interested on the existence and uniqueness of solution for equation (10), we refer to

7 the work of Kruzhkov (30).

8 Units and model structure between 1D and 2D are compared in the table 1:

9 The model described by the equation (10) is a direct extension in two dimensions of the 10 LWR model. However, several differences remain in the expression of the flux which make the 11 modeling problem more complex. To be valid, a first assumption is that $2 \mathrm{D}$ models have to describe 12 a road network dense enough such that the flux, the velocity and the density can be defined at 13 any point of the plane as an extension of the road nearby. The flux in 2D models is a vector 14 and without loss of generality, we can split it into a flux magnitude $m$ and a normalized vector 15 of direction $\vec{d}_{\theta}$ such that $\vec{\Phi}(t, x, y, \rho)=m(t, x, y, \rho) \vec{d}_{\theta}(t, x, y, \rho)$. We can notice that the notion of 


\begin{tabular}{|l|c|c|}
\hline & 1D model & 2D model \\
\hline density & {$[\rho]=v e h / m$ (scalar) } & {$[\rho]=v e h / m^{2}$ (scalar) } \\
velocity & {$[v]=m / s$ (scalar) } & {$[\vec{v}]=m / s$ (vector) } \\
flux & {$[\Phi=v \rho]=$ veh $/ s$ (scalar) } & {$[\vec{\Phi}=\vec{v} \rho]=v e h / s / m$ (vector) } \\
equation & $\partial_{t} \rho+\partial_{x} \Phi(\rho)=0$ & $\partial_{t} \rho+\nabla \cdot \vec{\Phi}(t, x, y, \rho)=0$ \\
\hline
\end{tabular}

TABLE 1 Comparison of model structure (unit are veh for vehicle, $m$ for space and $s$ for time.)

1 direction does not exist in one dimension, and a specific attention should be paid to introduce 2 the direction in the 2D model. The flux is defined as the product of the density and the velocity

$3 \vec{\Phi}(t, x, y, \rho)=\vec{v}(t, x, y, \rho) \rho(t, x, y)$. Thus in two dimensions, the velocity and the flux direction 4 coincide. In the following we will refer to the velocity or flux direction to discuss either of them. 5 The case where the flux (or velocity) function depends on time, space and density corresponds to the most general case. In the following, we investigate if each dependency is justified by traffic observations or if some of them could be relaxed.

\section{Derivation of the 2D flux magnitude}

In this section, we discuss the relevance of the dependency of the flux magnitude w.r.t. time, space and density.

First, we investigate the dependency of the flux magnitude w.r.t. the density. In one dimensional models, the velocity is defined as a decreasing function of the density, higher the density on the road, lower is the velocity. This idea seems consistent with the representation of a 2D model. Thus, we consider the Greenshields fundamental diagram as starting point to the definition of the flux magnitude in 2D. The expression of the velocity equation (4) depends on the density and also of two parameters: the maximum speed $v_{\max }$ and the maximum density $\rho_{\max }$.

The maximal speed $v_{\max }$ could vary with respect to time, hence enforcing a time dependence of the flux. However, for simplicity we will not consider time dependence in this paper.

Finally, it is possible to have a space dependency if the parameters $v_{\max }$ or $\rho_{\max }$ change along the road considered. For example, these modifications occur at the point of the road where the speed limit or the number of lane of the road change. However, variations of parameters require specific models and involve a more complex computation of the flux. In 2D model, vehicles run on an abstraction of the physical network in two dimensions. Thus the notion of capacity and speed limit are different and depend of an interpolation of the underlying road network. Furthermore, the scale considered is in general more large. If the parameters $v_{\max }$ and $\rho_{\max }$ have even more reason to change in $2 \mathrm{D}$ model, the spatial variation of these parameters could be ubiquitous. Thus, it seems essential to develop a precise and accurate method to take into account these variations. As this problem could be very complex to handle, we choose to leave this study for future work and we do not consider space dependencies for the magnitude in the following. This is equivalent to say that every roads of the network considered are homogeneous with the same capacity and speed limit.

\section{Variable dependencies of flux direction}

In this section, we discuss the relevance of the dependency of the flux direction with respect to time, space and density. Contrary to the magnitude dependency, only few comparison can be done with existing idea in one dimension as the notion of direction needs to be explicit only in 2D mod- 
1 els. Thus, we suggest three ideas regarding what could impact the flux direction.

2 First, the flux direction should depend on the road of the underlying network. Indeed, we aim to

3 design a model which allows the flux to go only in physically possible directions. That is to say

4 vehicles should not be able to move to a direction where there are no roads. Thus, we assumed that

5 the velocity direction depends on the space variables.

6 A second idea is that the flux direction depends on the individual wishes of drivers. In reality, the

7 flux direction is directly linked to the trajectory of each individual vehicle. However in practice,

8 macroscopic models are not able to keep track of individual drivers but we can still imagine that

9 the direction of the flux could be linked to a global average trajectory. Thus, the flux direction may

10 include dependency in the time and spatial coordinates. Indeed, the dependency of the flux direc-

11 tion with respect to the space is necessary in order to describe the trajectory. The time dependency

12 allows this trajectory to change during the simulation: for example, most of drivers may join the

13 city center in the morning and return in the suburb in the evening. We choose to not introduce

14 these dependency in the model as we would like to have a model that does not depend on external 15 data feed in real time.

16 Another idea is the fact that drivers may adapt their direction according to the local condition of 17 traffic. For example, drivers could change their planned itinerary in order to bypass a congestion.

18 This may lead to a dependency of the flux direction with the density. We choose to not keep this

19 dependency in the model.

20 The 2D model for traffic considered

The model considered is based on a two dimensional conservation law as equation (10). We recall that the flux function can be split in a magnitude part

$$
m(\rho):\left[0, \rho_{\max }\right] \rightarrow\left[0, \Phi_{\max }\right]=\rho \underbrace{\left(1-\frac{\rho}{\rho_{\max }}\right) v_{\max }}_{\text {velocity norm }}
$$

and a direction vector

$$
\vec{d}_{\theta}(x, y): \Omega \rightarrow \mathbb{R}^{2}=\left(\begin{array}{c}
\cos (\theta(x, y)) \\
\sin (\theta(x, y))
\end{array}\right) .
$$

21 with $\theta$ the angle between the x-axis and the vector direction of the flux. Then, the flux can be defined: $\vec{\Phi}(\rho(t, x, y), \theta(x, y))=m\left(\rho(t, x, y) \vec{d}_{\theta}(x, y)\right.$. The equation considered for the model is:

$$
\frac{\partial \rho(t, x, y)}{\partial t}+\frac{\partial m(\rho(t, x, y)) \cos (\theta(x, y))}{\partial x}+\frac{\partial m(\rho(t, x, y)) \sin (\theta(x, y))}{\partial y}=0
$$

The expression of $m$ is given by the Greenshields fundamental diagram, Figure 1(b). An expression is also needed for the direction of the flux in order to be able to make prediction of the density propagation. Thus, we suggest in the next section a way to estimate the function $d_{\theta}$ using as much as possible the information we have on the physical network.

\section{VELOCITY DIRECTION FIELD DESIGN FROM THE PHYSICAL NETWORK Description of the method}

The aim of this section is to suggest one possible way to construct the function $\vec{d}_{\theta}$ using only the topology of the road network. We have seen that for any point $(x, y) \in \Omega$, we need to define the 
1 direction of the flux $\vec{d}_{\theta(x, y)}$. A network is a set of roads in which the link represents the roads and 2 the nodes the junctions.

3 Lets $q \in\{1, \ldots, Q\}$ be the set of roads of the network with $\mathrm{Q}$ the number of roads. Each road $q$ 4 is described thanks to a parametric curve $\Psi^{q}: s \in[0,1] \rightarrow\left(\Psi_{1}^{q}(s), \Psi_{2}^{q}(s)\right)$ that follows the road 5 curvature from the initial point on the road, $\Psi^{q}(0)=\left(x_{d}, y_{d}\right)$, to a final point, $\Psi^{q}(1)=\left(x_{f}, y_{f}\right)$. 6 For all points $s$ of these parametric curves, we denote by $\vec{\tau} q\left(\Psi_{1}^{q}(s), \Psi_{2}^{q}(s)\right)$ the tangent vector of the 7 curve which represents also the direction of the road. These notations are represented in Figure 2.

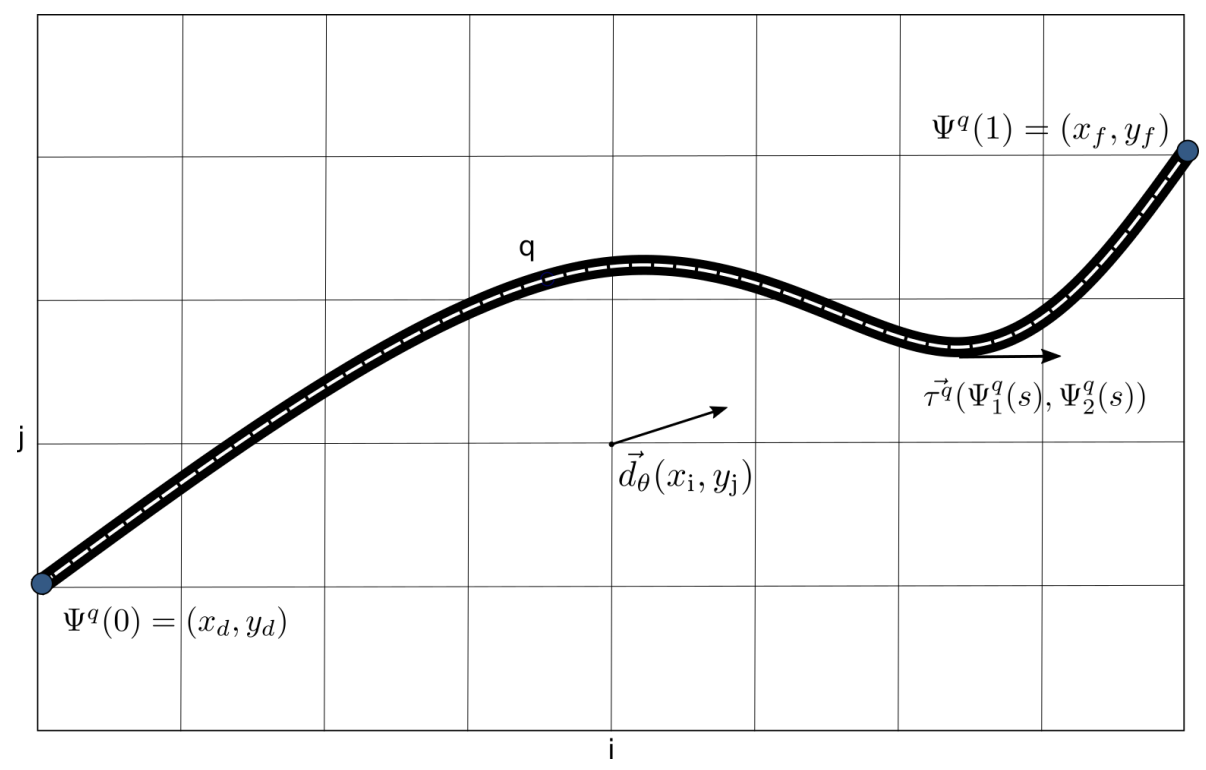

FIGURE 2 Representation of the road $q$ and his vector tangent $\vec{\tau}^{q}$

Let consider a discretisation of the space where $I$ and $J$ are respectively the number of discrete interval for the $\mathrm{x}$-axis and the $\mathrm{y}$-axis. For a given point $\left(x_{i}, y_{j}\right)_{(i, j) \in\{1, \ldots, I\} \times\{1, \ldots, J\}}$, let $\vec{d}_{\theta}\left(x_{i}, y_{j}\right)$ be the directional vector for which the angle with the $\mathrm{x}$ axis and the vector is $\theta\left(x_{i}, y_{j}\right)$ such that:

$$
\vec{d}_{\theta}\left(x_{i}, y_{j}\right)=\left(\begin{array}{c}
\cos \left(\theta\left(x_{i}, y_{j}\right)\right) \\
\sin \left(\theta\left(x_{i}, y_{j}\right)\right)
\end{array}\right) .
$$

13 To estimate the vector $\vec{d}_{\theta}$, we use a spatial interpolation method called Inverse Distance Weighting. 14 The expression of the estimator is given in equation (14):

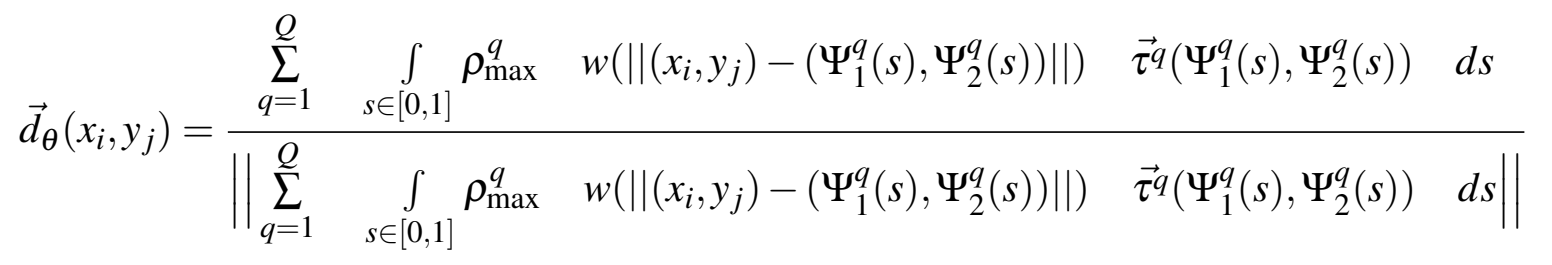

Thereby, the velocity direction $\vec{d}_{\theta}\left(x_{i}, y_{j}\right)$ is estimated as a linear combination of the direction $\vec{\tau}^{q}$ of every road $q \in\{1, \ldots, Q\}$ of the network. The weight coefficient of each road direction depends first on the distance between the road and the point $\left(x_{i}, y_{j}\right)$. This dependency is evaluated with the 
weight function $w: \mathbb{R}^{+} \rightarrow \mathbb{R}^{+}$strictly decreasing. The weight function considered in the following is:

$$
w: X \rightarrow e^{-\beta X} \quad \text { with } \quad \beta>0 .
$$

1 We choose the exponential weights in order to give greater weight to close roads, w.r.t. far away 2 ones. Furthermore, the weight coefficient can also depend of the capacity of the road $\rho_{\max }$ as roads 3 with higher capacity can hold more drivers.

\section{Example}

5 In the section, we present the result of the estimation of a velocity field for an artificial network

6 using the method described above.

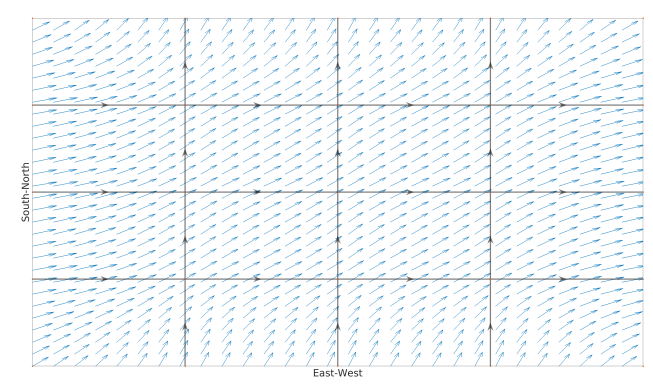

(a) $\beta=10$

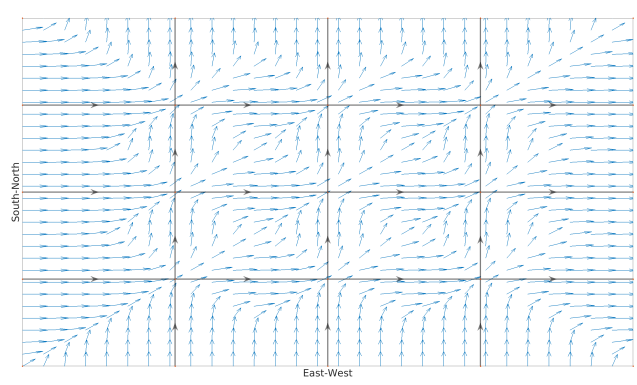

(b) $\beta=50$

FIGURE 3 Estimation of the velocity direction for a $5 \times 5$ Manhattan grid

$7 \quad$ Figure 3(a) and 3(b) represent the result of the estimation of the velocity direction for 8 respectively $\beta=10$ and $\beta=50$. When the parameter $\beta=10$, the direction field $\vec{d}_{\theta}$ gives only the

9 global direction of the road network whereas when $\beta=50$, the direction field describes more the

10 local direction of the road network. 

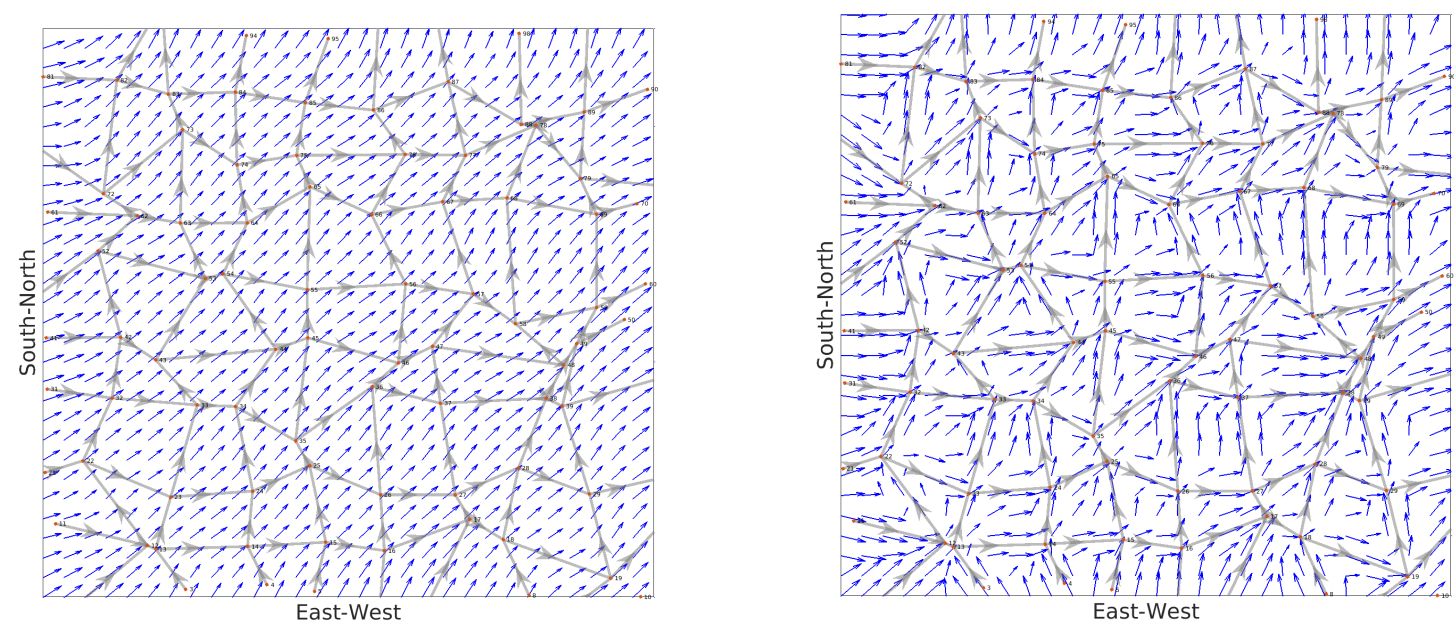

FIGURE 4 Estimation of the velocity direction for a less regular Manhattan grid for $\beta=20$ (left) and $\beta=100$ (right)

In order to test how adaptable is the method suggested, we considered as well, a Manhattan 2 grid for which the position of each nodes have been slightly modified. An example for two different 3 values of $\beta$ is presented in Figure 4.

4 NUMERICAL METHOD FOR SIMULATION

5 Choice of the method

6 This section focuses on the description of the simulation method for equation (12). The literature 7 regarding numerical methods for the two dimensional conservation law is quite broad. However, 8 only few of these studies include the case of flux function with space dependency. In Lie (31), the 9 authors consider the case of quasilinear hyperbolic equation: $\frac{\partial u}{\partial t}+U(x, y) \frac{\partial f(u)}{\partial x}+V(x, y) \frac{\partial g(u)}{\partial y}=0$. 10 It is possible to compute a stable approximation of solution of this equation using dimensional 11 splitting under the assumption that the function $U$ and $V$ are Lipschitz continuous and bounded. We 12 notice that it is possible to expand the derivative of equation (12) in order to reveal the quasilinear term and the source term: $\forall t \in \mathbb{R}^{+}, \forall(x, y) \in \Omega$,

$$
\frac{\partial \rho}{\partial t}+\underbrace{\cos (\theta(x, y)) \frac{\partial m(\rho)}{\partial x}+\sin (\theta(x, y)) \frac{\partial m(\rho)}{\partial y}}_{\text {Quasilinear }}=\underbrace{-m(\rho) *\left(\frac{\partial \cos (\theta(x, y))}{\partial x}+\frac{\partial \sin (\theta(x, y))}{\partial y}\right)}_{\text {Source }}
$$

14 The principle of splitting method is to compute separately the different term of the equation. Thus,

15

16 the formulation given by the equation (15) allows to modify the problem into the computation of an equation with a quasilinear term and an equation with a source term. The function $\cos (\theta(.,)$. and $\sin (\theta(.,)$.$) are bounded and are also Lipschitz continuous on condition that the function \theta$ is smooth. For the computation of the source term, we use the operator splitting method as it is presented in Toro (32) and Gosse (33). 
1 Description of splitting methods

2 Splitting methods have been considered first by Godunov (34) but have been formally introduced 3 by Strang (35). The concept of splitting is to divide the computation of the different terms of the 4 equation in several numerical steps. For the general case, this method is called operator splitting. 5 When the equation is divided with the derivative along different coordinates, it is called dimen6 sional splitting.

7 Let $i, j \in[1, \ldots, I] *[1, \ldots, J]$ be the discretization of the space and $n$ the index for the time step. 8 The iteration process for the computation of one time step is divided in three parts. First, we compute the propagation with respect to the first dimension. Then, we compute the propagation with respect to the second dimension using the updated density. Finally, we add the effect of the source 11 term.

$$
\begin{gathered}
\rho_{\mathrm{i}, \mathrm{j}}^{*}=\rho_{\mathrm{i}, \mathrm{j}}^{\mathrm{n}}-\cos \left(\theta_{\mathrm{i}, \mathrm{j}}\right) \frac{\Delta t}{\Delta x}\left(F_{\mathrm{i}+\frac{1}{2}, \mathrm{j}}^{n}-F_{\mathrm{i}-\frac{1}{2}, \mathrm{j}}^{n}\right) \\
\rho_{\mathrm{i}, \mathrm{j}}^{* *}=\rho_{\mathrm{i}, \mathrm{j}}^{*}-\sin \left(\theta_{\mathrm{i}, \mathrm{j}}\right) \frac{\Delta t}{\Delta y}\left(F_{\mathrm{i}, \mathrm{j}+\frac{1}{2}}^{\mathrm{n}}-F_{\mathrm{i}, \mathrm{j}-\frac{1}{2}}^{\mathrm{n}}\right) \\
\rho_{\mathrm{i}, \mathrm{j}}^{\mathrm{n}+1}=\rho_{\mathrm{i}, \mathrm{j}}^{* *}-m\left(\rho^{* *}\right)\left(\frac{\Delta t}{\Delta x}\left(\cos \left(\theta_{\mathrm{i}+\frac{1}{2}, \mathrm{j}}\right)-\cos \left(\theta_{\mathrm{i}-\frac{1}{2}, \mathrm{j}}\right)\right)+\frac{\Delta t}{\Delta y}\left(\sin \left(\theta_{\mathrm{i}, \mathrm{j}+\frac{1}{2}}\right)-\sin \left(\theta_{\mathrm{i}, \mathrm{j}-\frac{1}{2}}\right)\right)\right)
\end{gathered}
$$

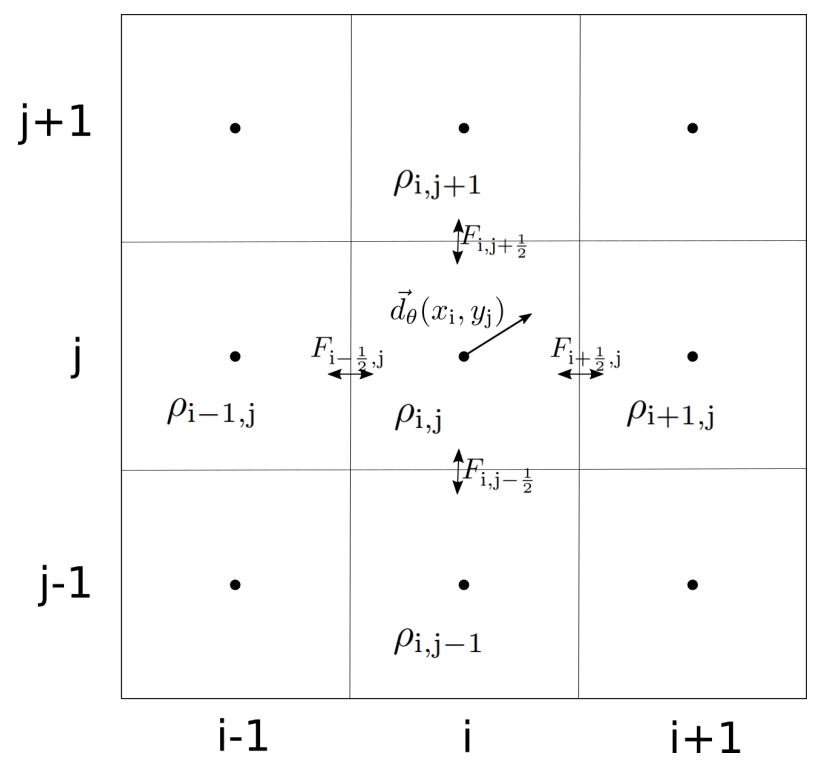

FIGURE 5 Spatial discretisation for the numerical method

15

For the notation, $F_{i+\frac{1}{2}, \mathrm{j}}^{n}$ corresponds to the Godunov flux between the cell as we can see in 16 Figure 5. To recall, the Godunov flux between a cell of density $u$ to an adjacent cell of density $v$, 17 for a the function $\Phi$ of the FD, can be written as follows:

$$
F(u, v)=\left\{\begin{array}{l}
\min (\Phi(u), \Phi(v)), \quad u<v \\
\Phi(u), \quad v \leq u \leq \rho_{\text {crit }} \\
\Phi(v), \quad \rho_{\text {crit }} \leq v \leq u \\
\Phi_{\max }, \quad v \leq \rho_{\text {crit }} \leq u
\end{array}\right.
$$

18 The boundary condition are treated by using ghost-cells. 
1 Algorithm

2 In this part, we describe the numerical implementation of the previous methods. The algorithm is

3 shown in Algorithm 1.

\section{Algorithm $12 \mathrm{D}$ model simulation}

1. 1: Define the parameters: $v_{\max }$ the maximum velocity, $\rho_{\max }$ the maximum density, $T_{\max }$ the time horizon, the constant coefficient CFL, $d x$ and $d y$ the spatial discretisation and $I$ and $J$ the number of discrete interval of the spatial discretisation.

2: Define the time step $d t=c f l * \min (d x, d y) / V_{\max }$

3: Import or create the road network of the study case

4: Compute the velocity field $d_{\theta} \quad \triangleright$ Using the estimation method

5: Initialize the density $\rho$ to a given value, the current time $t$ to zero.

6: while $t<T_{\max }$ do

7: $\quad$ Evalue the density at the next time step using the function Propagation_2D

8: $\quad$ function PROPAGATION_2D $\left(\rho^{\mathrm{n}}, d t, d x, d y, I, J, v_{\max }, \rho_{\max }, d_{\theta}\right)$

9: $\quad$ Define the intermediate densities $\rho^{*}$ and $\rho^{* *}$

10: $\quad$ for $i=1$ to $I$ and $j=1$ to $J$ do

11: $\quad$ Update of $\rho^{*}$ with flux along the $\mathrm{x}$ dimension computed with $\rho^{\mathrm{n}}$

12: $\quad$ end for

13: $\quad$ for $i=1$ to $I$ and $j=1$ to $J$ do

14: $\quad$ Update of $\rho^{* *}$ with flux along the y dimension computed with $\rho^{*}$

15: $\quad$ end for

16: $\quad$ for $i=1$ to $I$ and $j=1$ to $J$ do

17: $\quad$ Update of the density value $\rho^{\mathrm{n}+1}$ due to the source term computed with $\rho^{* *}$

18: $\quad$ end for

19: $\quad$ end function

20: $\quad \mathrm{t}=\mathrm{t}+\mathrm{dt}$

21: end while

\section{SIMULATION RESULTS ON AN ARTIFICIAL MANHATTAN GRID}

5 In this section, we analyze the results of the simulations of the model presented for two scenarios.

6 The scenarios represent the same network and thus have an identical estimation of the velocity

7 direction $\vec{d}_{\theta}$. The network considered is build as a Manhattan grid on a square domain where every

8 road taken into account is oriented towards the North-East. The network and the velocity field can

9 be seen in Figure 6. 


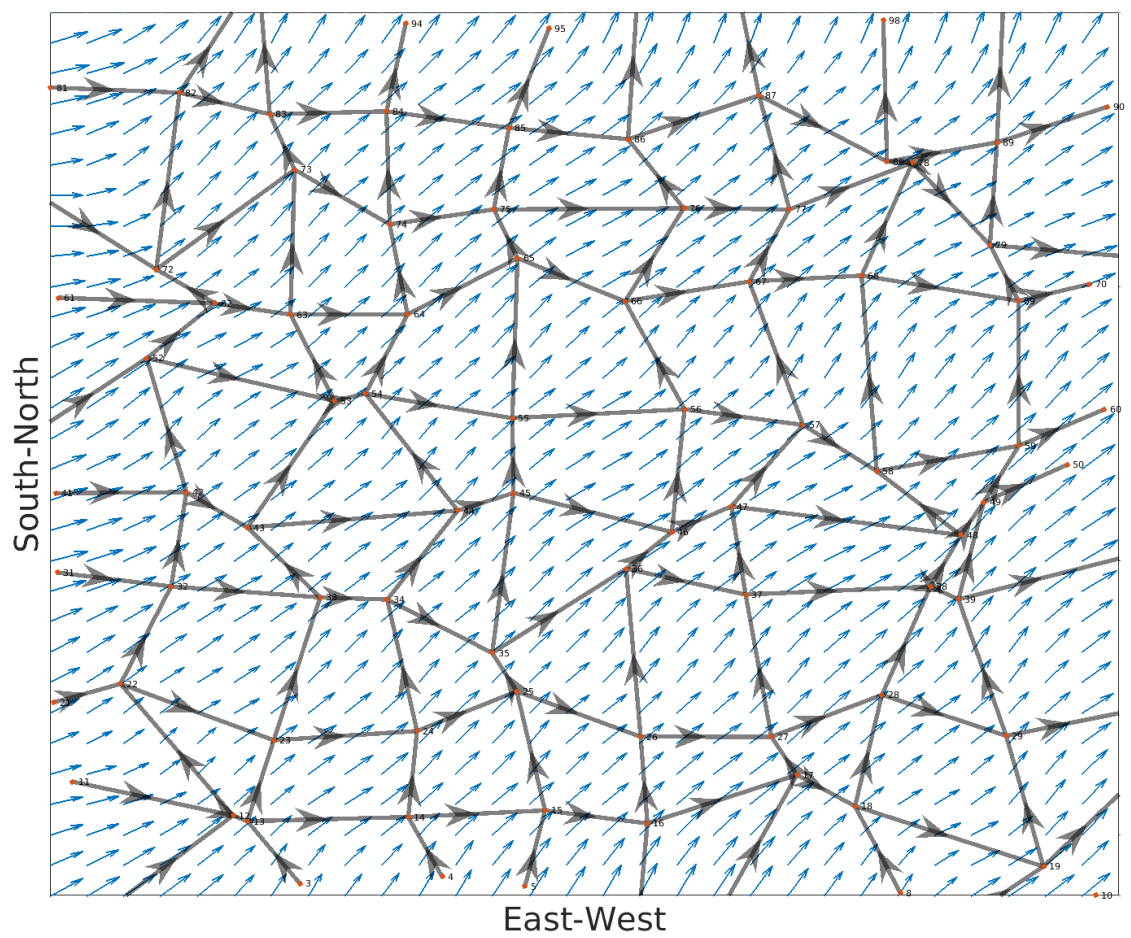

FIGURE 6 Network considered for the scenario (in black) and velocity direction estimated (in blue) for a parameter $\beta=20$

\section{Forward propagation on an artificial Manhattan grid}

2 In this section, we present the results of the simulations for an initial condition given by equation 3 (17):

$$
\rho_{0}(x, y)=\left\{\begin{array}{ll}
\frac{1}{2} \rho_{\max } & \text { if } \frac{1}{8} L_{\mathrm{x}} \leq x \leq \frac{4}{8} L_{\mathrm{x}} \\
0 & \text { otherwise }
\end{array} \text { and } \quad \frac{1}{8} L_{\mathrm{y}} \leq y \leq \frac{4}{8} L_{\mathrm{y}}\right.
$$

4 where $L_{\mathrm{x}}$ and $L_{\mathrm{y}}$ are respectively the length of the domain along the x-axis and the y-axis. In Figure 57 , we show the results for different times. 

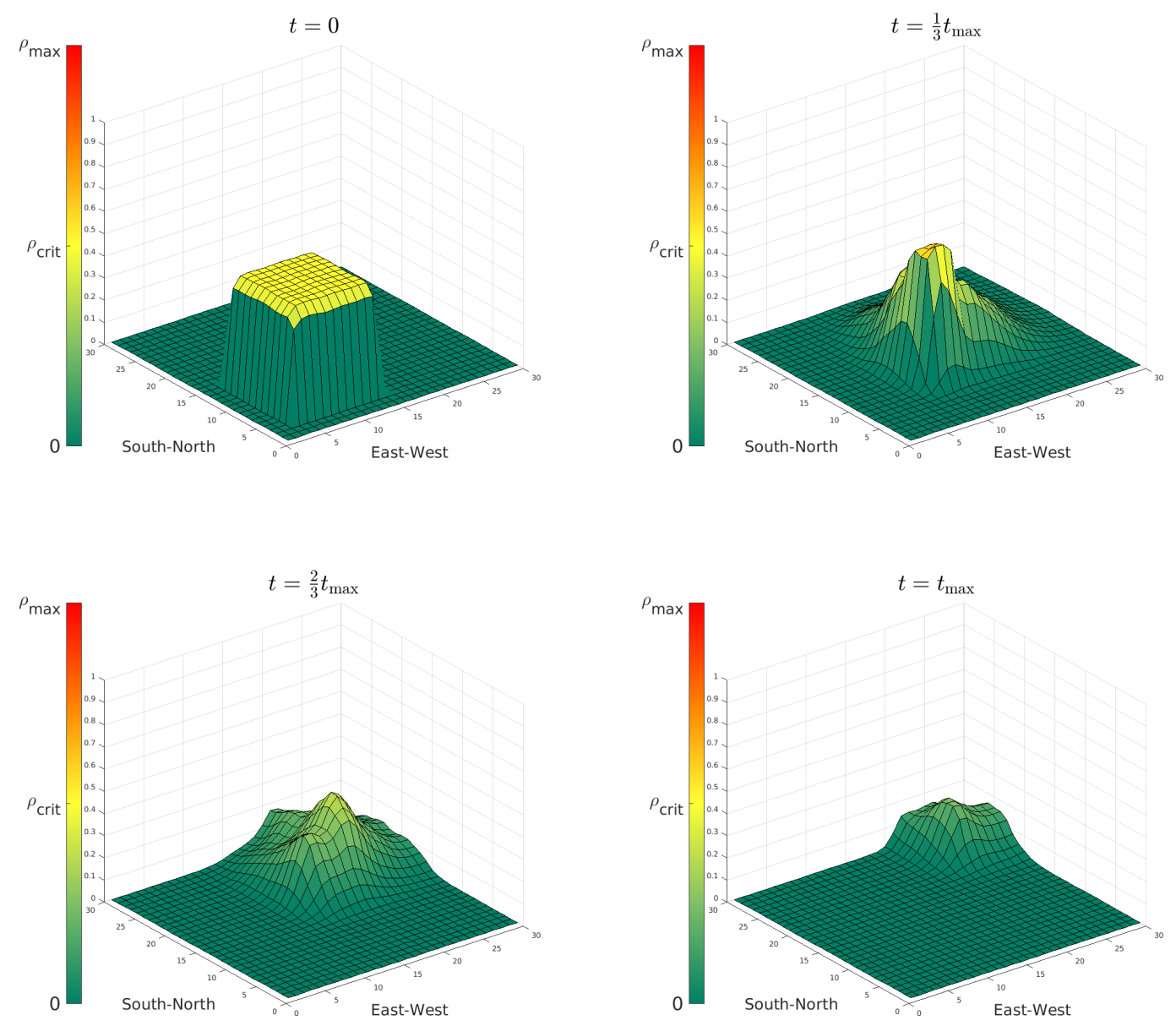

FIGURE 7 Simulation results at different times of $2 D$ model with initial traffic state given by equation (17)

1 First, we can remark that at the initial time, the initial conditions generate two main dis2 continuities. On the North-East, the discontinuity corresponds to a rarefaction wave whereas in the 3 South-West it is a shock wave. The shape of the shock wave is globally preserved.

\section{Backward propagation and comparison with the CTM model}

5 In this section, we present the results of the simulations for an initial condition given by equation 6 (18):

$$
\rho_{0}(x, y)= \begin{cases}\rho_{\max } & \text { if } \frac{2}{3} L_{\mathrm{x}} \leq x \text { and } \frac{2}{3} L_{\mathrm{y}} \leq y \\ \frac{9}{16} \rho_{\max } & \text { otherwise }\end{cases}
$$

7 The results at different times are given in Figure 8: 

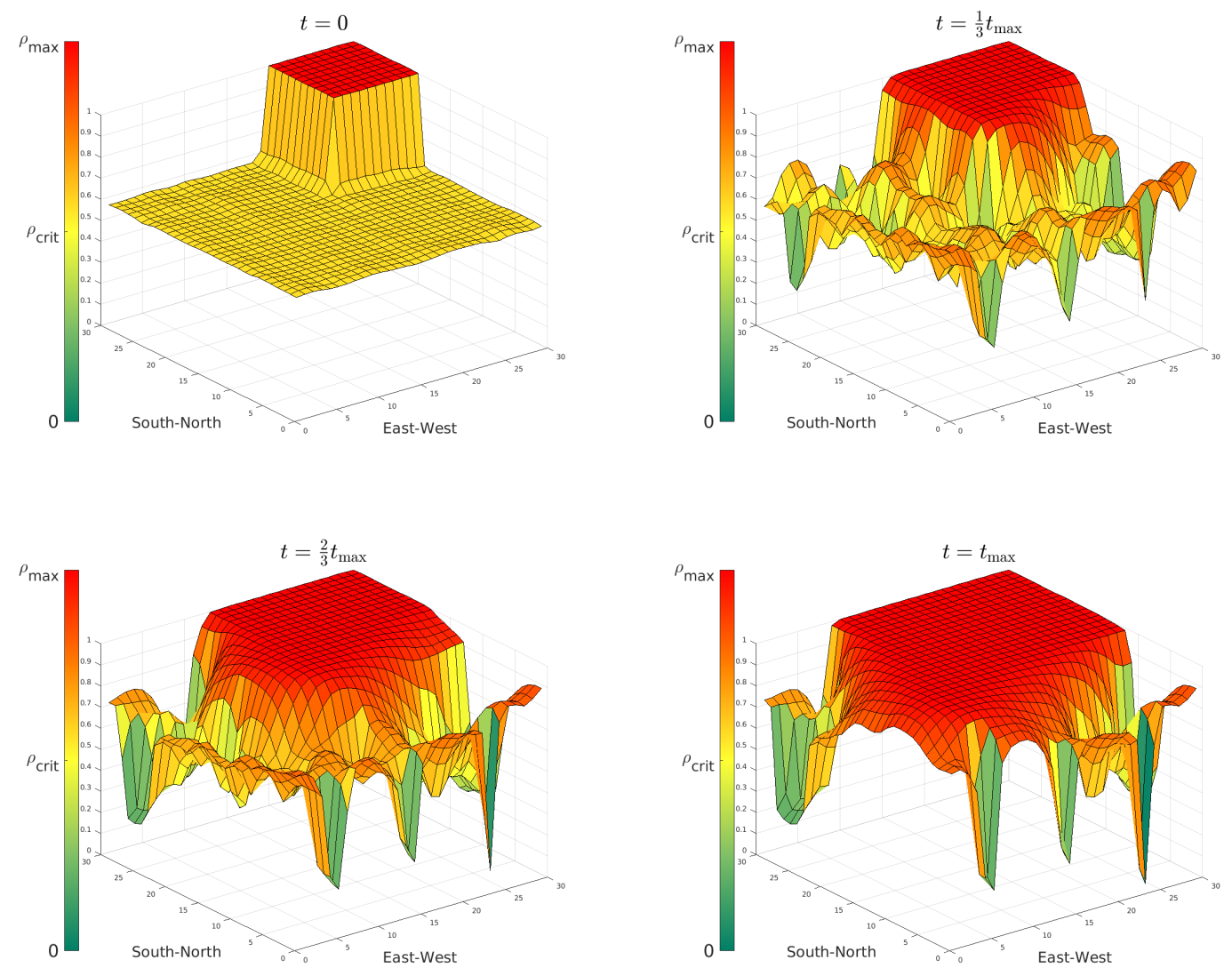

FIGURE 8 Simulation results at different times of $2 D$ model with initial traffic state given by equation (18)

1 We can observe how the density over all the network raises and the network is quickly 2 filled.

3 We have also simulated in parallel a Cell Transmission Model Daganzo (5) Coclite et al. (6) on the 4 same network and with the same initial condition, for comparison. The results of simulation are 5 presented in Figure 9: 

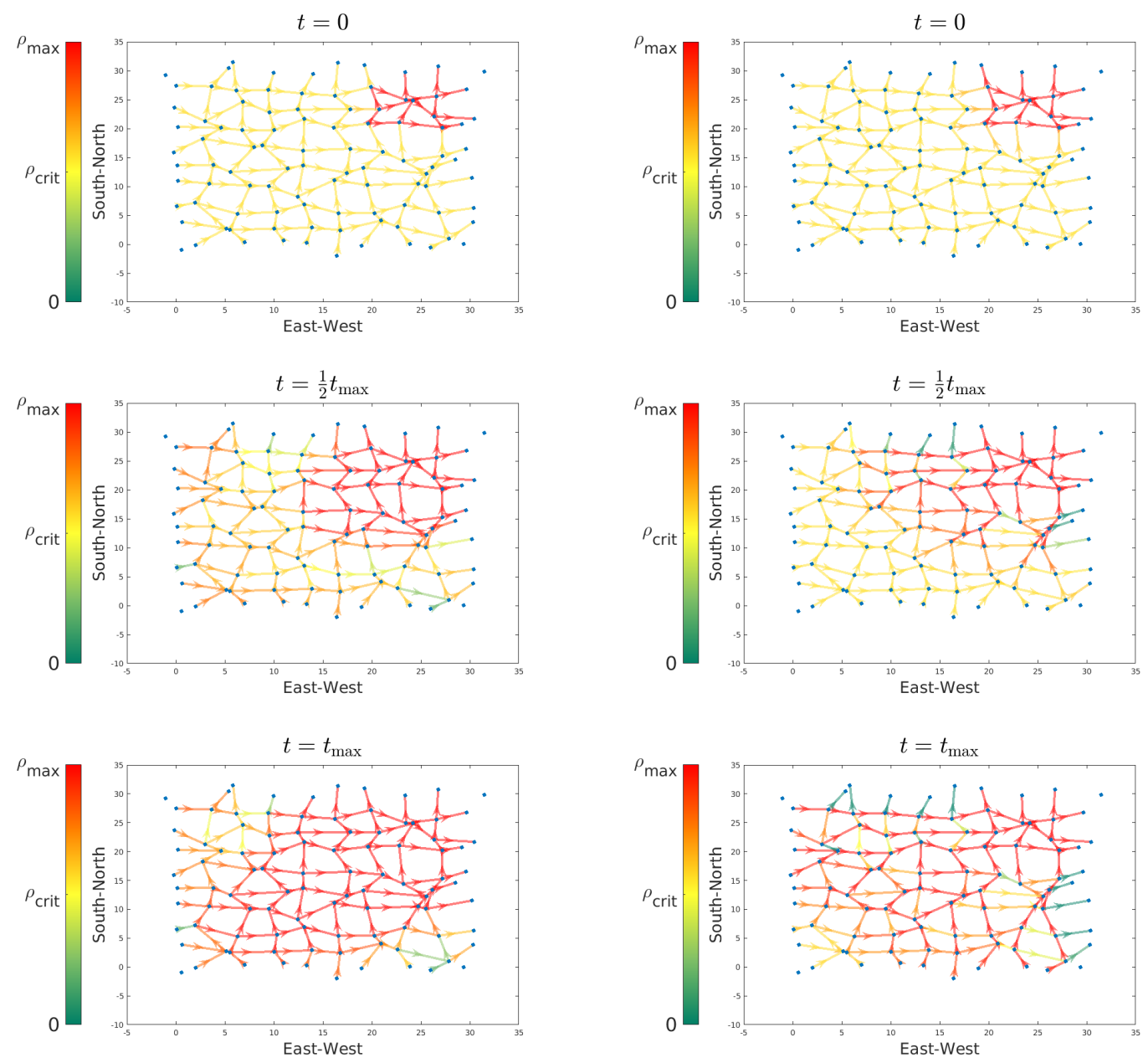

FIGURE 9 Comparison of simulation between 2D model (left) and CTM (right) for an initial traffic state given by equation (18)

We represent both simulation at the graph level. For the two dimensional model, the rep2 resentation is only visual. Indeed, we have just done the projection of the two dimensional grid 3 coloring on the graph. Both in the 2D model and in the junction model, the wave propagate back4 ward as expected with approximately the same speed of propagation.

\section{CONCLUSION AND FUTURE WORK}

6 This article is an attempt to extend the LWR model in a two dimensional setting. Contrary to the 7 one dimensional case, we underline the fact that we need to complete the definition of the flux 8 function by additional information. In particular, we focus on the flux direction and we suggest a 9 way to estimate it from the physical network. Finally, we present results of simulations which allow to make comparison with the features observed on the LWR model in one dimension. Future work 
1 will involve the extension of the model to heterogeneous networks, i.e., capacity and speed limit

2 variations w.r.t. space and time and the possibility to describe simultaneously flow in different

3 direction. A direct extension of this model is the use of split ratios in the computation of the

4 velocity field.

\section{ACKNOWLEDGEMENTS}

6 This project has received funding from the European Research Council (ERC) under the European

7 Union's Horizon 2020 research and innovation programme (grant agreement 694209).

\section{REFERENCES}

[1] van Wageningen-Kessels, F., H. Van Lint, K. Vuik, and S. Hoogendoorn, Genealogy of traffic flow models. EURO Journal on Transportation and Logistics, Vol. 4, No. 4, 2014, pp. 445473.

[2] Greenshields, B. D., J. Thompson, H. Dickinson, and R. Swinton, The photographic method of studying traffic behavior. In Highway Research Board Proceedings, 1934, Vol. 13.

[3] Lighthill, M. J. and G. B. Whitham, On kinematic waves. II. A theory of traffic flow on long crowded roads. In Proceedings of the Royal Society of London A: Mathematical, Physical and Engineering Sciences, The Royal Society, 1955, Vol. 229, pp. 317-345.

[4] Richards, P. I., Shock waves on the highway. Operations research, Vol. 4, No. 1, 1956, pp. 42-51.

[5] Daganzo, C. F., The cell transmission model: A dynamic representation of highway traffic consistent with the hydrodynamic theory. Transportation Research Part B: Methodological, Vol. 28, No. 4, 1994, pp. 269-287.

[6] Coclite, G. M., M. Garavello, and B. Piccoli, Traffic flow on a road network. SIAM journal on mathematical analysis, Vol. 36, No. 6, 2005, pp. 1862-1886.

[7] Daganzo, C. F. and N. Geroliminis, An analytical approximation for the macroscopic fundamental diagram of urban traffic. Transportation Research Part B: Methodological, Vol. 42, No. 9, 2008, pp. 771-781.

[8] Geroliminis, N. and J. Sun, Properties of a well-defined macroscopic fundamental diagram for urban traffic. Transportation Research Part B: Methodological, Vol. 45, No. 3, 2011, pp. 605-617.

[9] Geroliminis, N. and C. F. Daganzo, Existence of urban-scale macroscopic fundamental diagrams: Some experimental findings. Transportation Research Part B: Methodological, Vol. 42, No. 9, 2008, pp. 759-770.

[10] Hajiahmadi, M., V. L. Knoop, B. De Schutter, and H. Hellendoorn, Optimal dynamic route guidance: A model predictive approach using the macroscopic fundamental diagram. In Intelligent Transportation Systems-(ITSC), 2013 16th International IEEE Conference on, IEEE, 2013, pp. 1022-1028.

[11] Leclercq, L., C. Parzani, V. L. Knoop, J. Amourette, and S. P. Hoogendoorn, Macroscopic traffic dynamics with heterogeneous route patterns. Transportation Research Procedia, Vol. 7 , 2015, pp. 631-650.

[12] Beckmann, M., A continuous model of transportation. Econometrica: Journal of the Econometric Society, 1952, pp. 643-660.

[13] Ho, H. and S. Wong, Two-dimensional continuum modeling approach to transportation prob- 
lems. Journal of Transportation Systems Engineering and Information Technology, Vol. 6, No. 6, 2006, pp. 53-68.

[14] Helbing, D., A fluid dynamic model for the movement of pedestrians. arXiv preprint cond-mat/9805213, 1998.

[15] Hughes, R. L., A continuum theory for the flow of pedestrians. Transportation Research Part B: Methodological, Vol. 36, No. 6, 2002, pp. 507-535.

[16] Jiang, Y.-q., P. Zhang, S. Wong, and R.-x. Liu, A higher-order macroscopic model for pedestrian flows. Physica A: Statistical Mechanics and its Applications, Vol. 389, No. 21, 2010, pp. 4623-4635.

[17] Colombo, R. M., M. Garavello, and M. Lécureux-Mercier, A class of nonlocal models for pedestrian traffic. Mathematical Models and Methods in Applied Sciences, Vol. 22, No. 04, 2012, p. 1150023.

[18] Twarogowska, M., P. Goatin, and R. Duvigneau, Comparative study of macroscopic pedestrian models. Transportation Research Procedia, Vol. 2, 2014, pp. 477-485.

[19] Kormanová, A. et al., A review on macroscopic pedestrian flow modelling. Acta Informatica Pragensia, Vol. 2, No. 2, 2014, pp. 39-50.

[20] Della Rossa, F., C. D'Angelo, and A. Quarteroni, A distributed model of traffic flows on extended regions. NHM, Vol. 5, No. 3, 2010, pp. 525-544.

[21] Jiang, Y., S. Wong, H. Ho, P. Zhang, R. Liu, and A. Sumalee, A dynamic traffic assignment model for a continuum transportation system. Transportation Research Part B: Methodological, Vol. 45, No. 2, 2011, pp. 343-363.

[22] Du, J., S. Wong, C.-W. Shu, T. Xiong, M. Zhang, and K. Choi, Revisiting JiangâÁŹs dynamic continuum model for urban cities. Transportation Research Part B: Methodological, Vol. 56, 2013, pp. 96-119.

[23] Jiang, Y.-Q., P.-J. Ma, and S.-G. Zhou, Macroscopic modeling approach to estimate trafficrelated emissions in urban areas. Transportation Research Part D: Transport and Environment, 2015.

[24] Romero Perez, L. and F. G. Benitez, Outline of Diffusion Advection in Traffic Flow Modeling. In Transportation Research Board 87th Annual Meeting, 2008, 08-1503.

[25] Saumtally, T., Modèles bidimensionnels de trafic. Ph.D. thesis, Université Paris-Est, 2012.

[26] Sossoe, K. S. and J.-P. Lebacque, Reactive Dynamic Assignment for a Bi-dimensional Traffic Flow Model. In International Conference on Systems Science, Springer, 2016, pp. 179-188.

[27] Chetverushkin, B., N. Churbanova, and M. Trapeznikova, Traffic Flow Simulation by 2D Macro- and Microscopic Models. In Latest Trends on Urban Planning Transportation, 2010.

[28] Sukhinova, A. B., M. A. Trapeznikova, B. N. Chetverushkin, and N. G. Churbanova, Two-dimensional macroscopic model of traffic flows. Mathematical models and computer simulations, Vol. 1, No. 6, 2009, p. 669.

[29] Lebacque, J. and M. Khoshyaran, First order macroscopic traffic flow models for networks in the context of dynamic assignment. Transportation Planning, 2004, pp. 119-140.

[30] Kruzhkov, S. N., First order quasilinear equations in several independent variables. Matematicheskii Sbornik, Vol. 123, No. 2, 1970, pp. 228-255.

[31] Lie, K.-A., A dimensional splitting method for quasilinear hyperbolic equations with variable coefficients. BIT Numerical Mathematics, Vol. 39, No. 4, 1999, pp. 683-700.

[32] Toro, E. F., Riemann solvers and numerical methods for fluid dynamics: a practical introduction. Springer Science \& Business Media, 2013. 
1 [33] Gosse, L., A two-dimensional version of the Godunov scheme for scalar balance laws. SIAM 2 Journal on Numerical Analysis, Vol. 52, No. 2, 2014, pp. 626-652.

3 [34] Godunov, S. K., A difference method for numerical calculation of discontinuous solutions of 4 the equations of hydrodynamics. Matematicheskii Sbornik, Vol. 89, No. 3, 1959, pp. 2715306.

6 [35] Strang, G., On the construction and comparison of difference schemes. SIAM Journal on $7 \quad$ Numerical Analysis, Vol. 5, No. 3, 1968, pp. 506-517. 